\title{
Isolation of new polyketide metabolites, linearolides A and B, from Streptomyces sp. RK95-74
}

\author{
Masashi Ueki $^{1}$, Naofumi Koshiro ${ }^{1,2}$, Harumi Aono ${ }^{1}$, Makoto Kawatani ${ }^{1}$, Masakazu Uramoto ${ }^{1}$, \\ Hisashi Kawasaki ${ }^{2}$ and Hiroyuki Osada ${ }^{1}$
}

\begin{abstract}
Although all Streptomyces strains are now thought to have 20-30 gene clusters for secondary metabolite biosynthesis, we cannot actually identify so many kinds of metabolites from one strain by conventional methods. Using Streptomyces sp. RK95-74, previously found as a cytotrienin producer, we searched new metabolites other than cytotrienin derivatives. Following the cultivation with new media and the peak-guided fractionation, we have found new compounds with new polyketide scaffold, named linearolides $\mathbf{A}$ and $\mathbf{B}$.
\end{abstract}

The Journal of Antibiotics (2013) 66, 333-337; doi:10.1038/ja.2013.19; published online 27 March 2013

Keywords: polyketide; Streptomyces

\section{INTRODUCTION}

Actinobacteria, most notably of Streptomyces, produce a wide variety of secondary metabolites useful for chemotherapy, such as antimicrobial, antitumor and immunosuppressive compounds. Indeed, owing to the ability to easily isolate and culture the microorganisms that are mainly distributed in soil, approximately two-thirds of all known antibiotics and half of all other bioactive metabolites have been isolated from Actinobacteria. ${ }^{1}$ Currently, the rate of new compound indentification from Actinobacteria is rapidly decreasing, however, previously undiscovered compounds have the possibility of being detected if more effective screening methods are used. ${ }^{2}$ These natural products are still recognized as a major source of clinical agents and chemotherapeutics. ${ }^{3}$

Over the past decade, the genome analyses of several Actinobacteria have been completed, following the initial genomic analysis of Streptomyces coelicolor $\mathrm{A} 3(2)^{4}$ and S. avermitilis. ${ }^{5}$ These genomes revealed that several families, Streptomycetaceae (Streptomyces and Kitasatospora), Micromonosporaceae (Salinispora and Micromonospora), and Psedonocardiaceae (Saccharopolyspora) in particular, presumably possess more than 20 gene clusters responsible for secondary metabolite biosynthesis. ${ }^{6}$ However, we cannot obtain such a various kind of metabolites from a single strain corresponding to a number of the gene clusters. The genetic capacity for secondary metabolite production is mostly cryptic, and the secondary metabolisms are not usually expressed under the conditions. ${ }^{7}$ Furthermore, studies often focus on only one specific metabolite and/or its derivatives from one strain, which were identified as active during a screening process aimed at finding new bioactive compounds. The gap between the potential hidden metabolites in the genomes and the actual metabolites produced through secondary metabolisms remains a major barrier in drugseeds discovery research. There is a continuing interest in the identification of new natural products with novel scaffolds or clinically relevant properties, as well as in the characterization of their biosynthesis, for the purpose of designing new natural product derivatives. $^{8}$

Streptomyces sp. RK95-74 has been found as a producer of cytotrienin A, a new member of ansatrienin antibiotics inducing apoptosis against leukemia cell lines. ${ }^{9,10}$ Draft genome sequence of strain RK95-74 revealed that there were more than 30 putative gene clusters for secondary metabolite biosynthesis in approximately 10.2 mega bases genome (unpublished data). However, we could not identify several metabolites such as nocardamin and proporphyrins other than cytotrienin derivatives under the condition for cytotrienin production. Understanding the actual roles of the biosynthetic gene clusters requires the identification of actual metabolites from this strain. To identify metabolites it is capable of producing, we cultivated strain RK95-74 under different media conditions and subsequently isolated the new secondary metabolites. This study represents the isolation of the new polyketide metabolites with novel scaffold, named linearolide A and B (Figure 1), from Streptomyces sp. RK95-74.

\section{RESULTS AND DISCUSSION}

New metabolites from Streptomyces sp. RK95-74

On the basis of the peak-guided fractionation, we identified two novel metabolites, one (1) from YMG culture extract, and the other (2) from PEC culture extract. The structures of these metabolites were elucidated using various instrumental analyses (Figure 2, Table 1).

${ }^{1}$ Antibiotics Laboratory, Chemical Biology Core Facility, RIKEN ASI, Wako, Japan and ${ }^{2}$ Department of Green and Sustainable Chemistry, Faculty of Engineering, Tokyo Denki University, Tokyo, Japan

Correspondence: Professor H Osada, Antibiotics Laboratory, Chemical Biology Core Facility, RIKEN ASI, 2-1 Hirosawa, Wako 351-0198, Japan.

E-mail: hisyo@postman.riken.go.jp

Received 7 November 2012; revised 25 January 2013; accepted 18 February 2013; published online 27 March 2013 


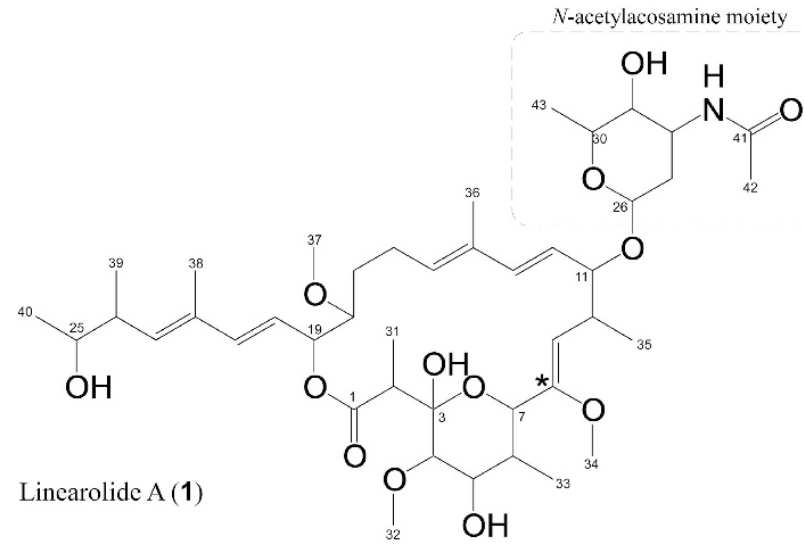

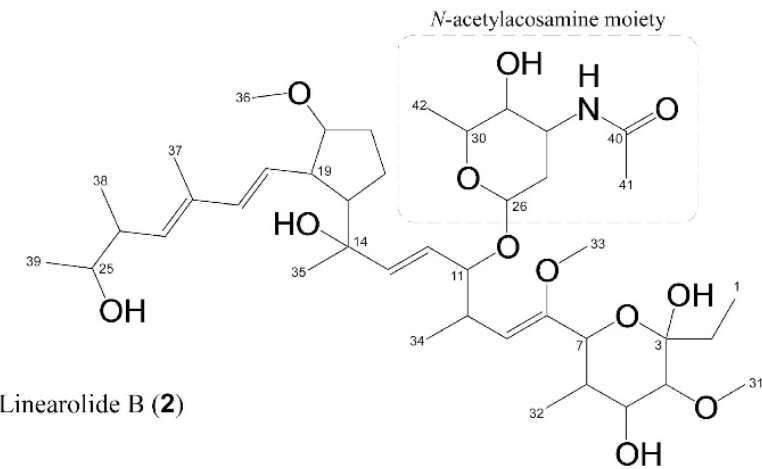

Figure 1 Structures of linearolides A (1) and B (2), isolated from strain RK95-74. An asterisk $\left({ }^{*}\right)$ indicates this configuration was not determined.

Compound 1 gave a pseudomolecular ion at $\mathrm{m} / \mathrm{z} 830.4635$ $[\mathrm{M}+\mathrm{Na}]^{+}$in HRESI-TOF-MS measurement, suggesting that its molecular formula is $\mathrm{C}_{43} \mathrm{H}_{69} \mathrm{NO}_{13}$ (calcd for $\mathrm{M}+\mathrm{Na}$ : 830.4667). UV and IR spectra of $\mathbf{1}$ in acetonitrile are shown in Figure $3 \mathrm{a}$ and Supplementary Figure S1A, respectively. Specific rotation $[\alpha]_{D}$ at $29.0^{\circ} \mathrm{C}$ of 1 in methanol was $+21.8(c=0.7)$. An inspection of the ${ }^{1} \mathrm{H}$ and ${ }^{13} \mathrm{C}$ NMR spectra of $\mathbf{1}$ in DEPT and HSQC experiments revealed the presence of 3 methyl groups bound to oxygen-bearing carbon or carbonyl (C-40, C-42, C-43), 4 secondary methyl groups (C-31, C-33, C-35, C-39), 2 allylic methyl groups (C-36, C-38), 3 methoxy groups (C-32, C-34, C-37), 11 oxygen-bearing carbons (C-3, C-4, C-5, C-7, C-11, C-18, C-19, C-25, C-26, C-29, C-30), $3 s p^{3}-$ methylenes (C-16, C-17, C-27), $7 s p^{2}$-methines (C-9, C-12, C-13, C-15, C-20, C-21, C-23), 2 quaternary $s p^{2}$ carbons (C-14, C-22), 2 carbonyls (C-1, C-41), and oxygen-bearing $s p^{2}$ carbon $(\mathrm{C}-8)$. The ${ }^{1} \mathrm{H}-$ ${ }^{1} \mathrm{H}$ COSY experiment of metabolite 1 led to five partial structure units, as shown by bold-faced lines in Figure 2. Further ${ }^{1} \mathrm{H}-{ }^{13} \mathrm{C}$ HMBC analysis, shown by arrows in Figure 2, revealed that a carbonyl at C-1 was bound to C-19 through an ester bond and connected to C-3 through C-2. Furthermore, 3 methoxy groups (C-32, C-34 and C-37) were bound to C-4, C-8 and C-18, respectively. The Egeometries of the olefins were deduced from large vicinal coupling constants between $\mathrm{H}-12$ and $\mathrm{H}-13(15.6 \mathrm{~Hz})$, and $\mathrm{H}-20$ and $\mathrm{H}-21$ $(15.5 \mathrm{~Hz})$. The other relative configurations were elucidated by $J$-based configuration analysis and NOESY experiment. Four key NOESY correlations for $\mathrm{H}-13 / \mathrm{H}-15, \mathrm{H}-12 / \mathrm{H}-36, \mathrm{H}-21 / \mathrm{H}-23$ and $\mathrm{H}-24 / \mathrm{H}-38$ suggested both these two methyl olefins were
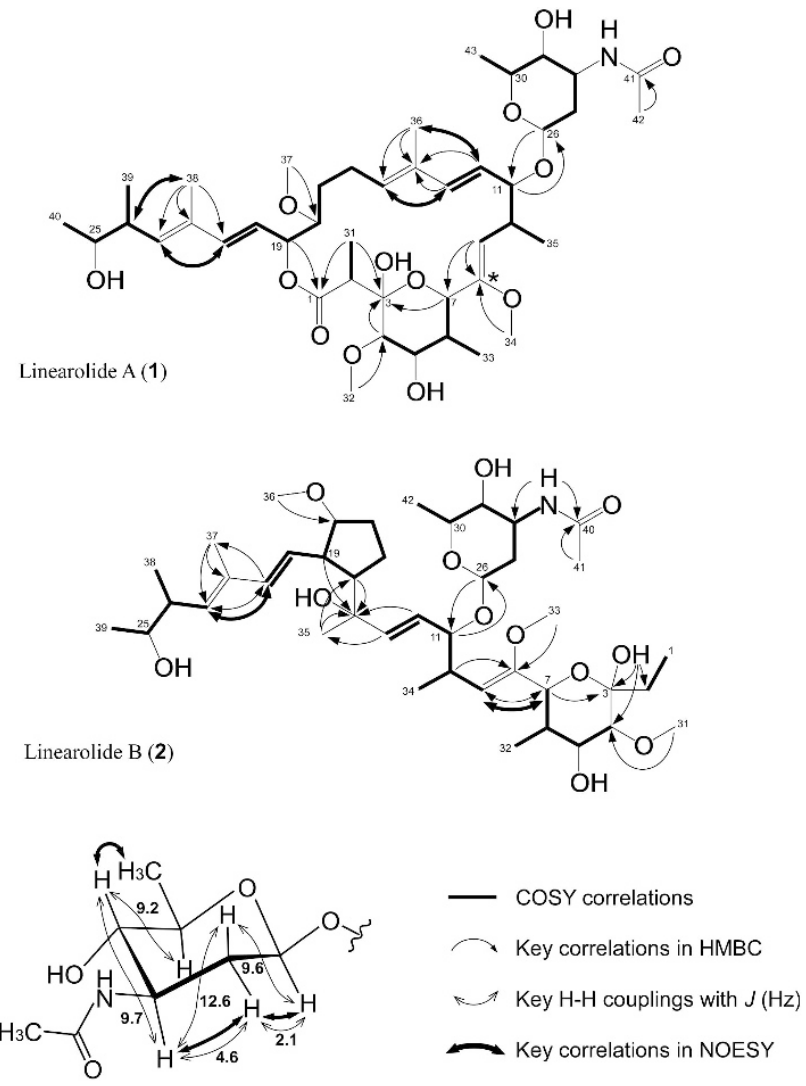

Relative structure of $\mathrm{N}$-acetylacosamine

Figure 2 NMR correlation of linearolides A (1), B (2), and N-acetylacosamine. An asterisk (*) indicates this configuration was not determined.

E configurations. The configuration of C-8 was not determined by NOESY because of no key correlation observed. However, the configuration of C-8 in 2 was determined to be $Z$, as described below. As 1 and 2 seemed to be biosynthesized through the same polyketide assembly line, the configuration of C-8 in 1 was expected to be identical with that in 2 as shown in Figures 1 and 2. This should be determined during the future work to elucidate the absolute structures of 1 and 2 . On the basis of $J$-based configuration and NOESY experiment, the relative structure of $N$-acetyl aminosugar was deduced to be $N$-acetylacosamine ${ }^{11}$ as shown in Figure 2. Lastly, a carbon C-7 was connected to C-3 through an ether bond, and to C-9 through C-8. The ether bond connections between C-26 and C-30/C11 suggested an $\mathrm{N}$-acetylacosamine moiety bound to the C-11 position, shown by a dotted rectangle in Figure 1. The connections between these units and the remaining functional groups were determined on the basis of key HMBC correlations summarized in Figure 2. Thus, the planar structure of $\mathbf{1}$ was elucidated as shown in Figure 1.

Compound 2 gave a pseudomolecular ion at $\mathrm{m} / \mathrm{z} 804.4866$ $[\mathrm{M}+\mathrm{Na}]^{+}$in HRESI-TOF-MS measurements, suggesting that its molecular formula is $\mathrm{C}_{42} \mathrm{H}_{71} \mathrm{NO}_{12}$ (calcd for $\mathrm{M}+\mathrm{Na}$ : 804.4874). UV and IR spectra of 2 in acetonitrile are shown in Figure $3 \mathrm{~b}$ and Supplementary Figure $S 1 B$, respectively. Specific rotation $[\alpha]_{D}$ at $29.0^{\circ} \mathrm{C}$ of 2 in methanol was $+85.0^{\circ}(c=0.1)$. In ${ }^{1} \mathrm{H}$ and ${ }^{13} \mathrm{C}$ NMR spectra of 2 with DEPT and HSQC analyses, a carbonyl signal (C-1 of 1) was disappeared and a methylene (C-2) was observed. The correlations from $3-\mathrm{OH}$ to $\mathrm{C}-2 / \mathrm{C}-3 / \mathrm{C}-4$ and from $\mathrm{H}-7$ to $\mathrm{C}-3$ in 
Table 1 NMR spectra data of linearolides A (1), and B (2)

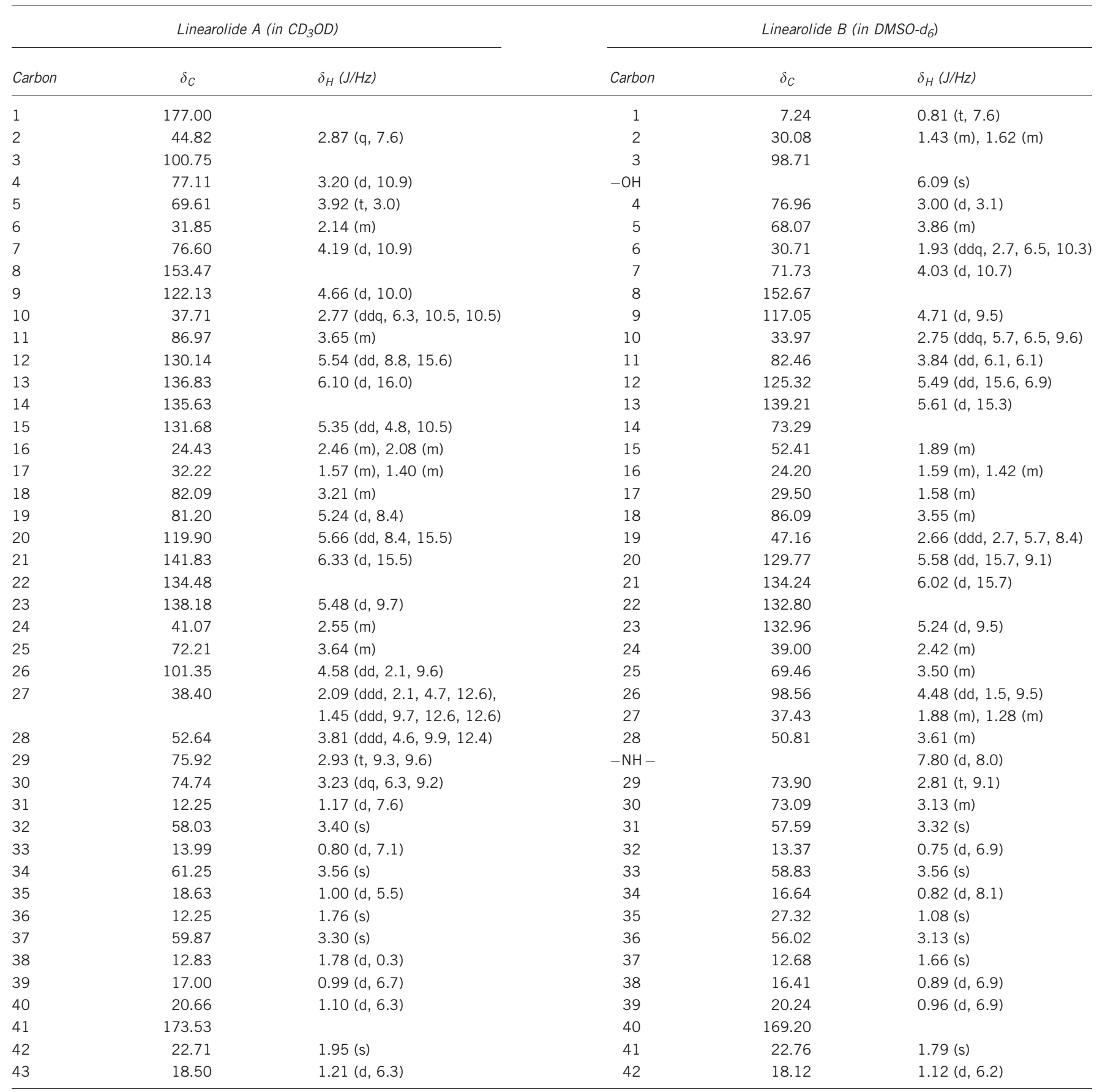

${ }^{1} \mathrm{H}-{ }^{13} \mathrm{C}$ HMBC and COSY correlations between C-4 and C-7 revealed an oxane ring moiety. Proton signals of other hydroxyl groups could not be assigned because they were broad and did not show any correlations. Almost all other signals of 2 were similar to those of 1, with the exception of non-existent oxygen attached to C-19 and a perceived oxygen-bearing carbon $\mathrm{C}-14$. The observed correlations between $\mathrm{C}-15$ and $\mathrm{C}-19$ in ${ }^{1} \mathrm{H}-{ }^{1} \mathrm{H}$ COSY and the connection between C-14 and C-15/C-19 in ${ }^{1} \mathrm{H}-{ }^{13} \mathrm{C}$ HMBC led to a methoxycyclopentane ring as shown in Figure 2. The configurations of olefins except C-8 were determined as well as those in 1 . The correlations between $\mathrm{H}-7$ and $\mathrm{H}-9$ in NOESY revealed that the olefin at C-8 was $Z$ form.
Furthermore, the correlations between a doublet amide signal $\left(\delta_{\mathrm{H}} 7.80\right)$ and $\mathrm{C}-40 / \mathrm{C}-28$, and methyl C-41 and C-40 revealed the attachment of $\mathrm{N}$-acetyl moiety to C-28. Therefore, the planar structure of 2 was elucidated as shown in Figure 1.

To our knowledge, this is the first report on the structures of $\mathbf{1}$ and 2, and we named them linearolide $\mathrm{A}$ and $\mathrm{B}$, respectively (Figure 1). Their absolute configurations, however, could not be elucidated because of the small amount of these metabolites. Interestingly, linearolide A (1) was obtained from YMG culture broth, while linearolide B (2) was not detected by LC/MS in YMG culture extract. Similarly, linearolide B (2) was obtained from 

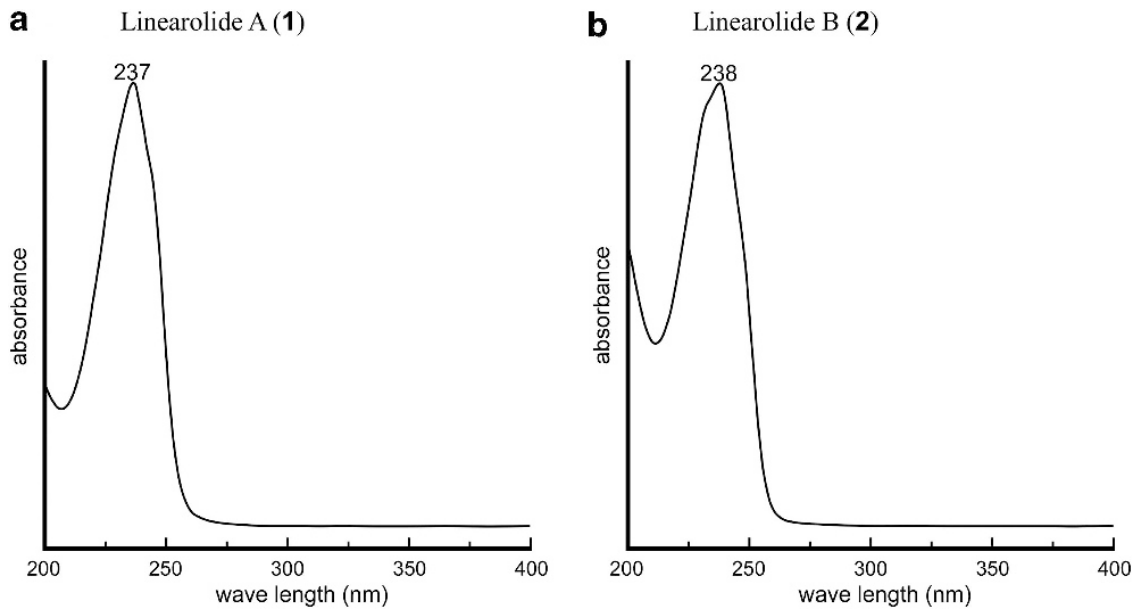

Figure 3 UV spectra of linearolides (a) A (1) and (b) B (2). $\lambda_{\max }(\mathrm{nm})$ were indicated on each spectrum.

PEC culture broth, and linearolide A (1) could not be detected in PEC extract. These findings suggested that the expression of an enzyme(s) involved in the formation of a macrocycle or an ether ring is regulated under culture conditions.

We hypothesize that both linearolides A and B are biosynthesized through the same polyketide assembly line, although post-PKS finalization to form a large macrocycle or small ether ring may be afforded by different enzymes. Through the identification and characterization of linearolide biosynthetic genes, we plan to create the new linearolide derivatives possessing biological activities in future studies.

\section{Biological properties of linearolides A and B}

Both compounds were tested for antimicrobial assay using the paper disc method and test microorganisms. No inhibitory zones were observed around paper discs containing $100 \mu \mathrm{g}$ of the compounds. Both compounds were also tested for cytotoxicity assay using several animal cell lines. As shown in Table 2, linearolides A inhibited the growth of HL-60 cells, and showed moderate cytotoxicity against other tested cells. Linearolide B showed no remarkable cytotoxicity. We speculate that the cyclization may be required for the biological activity. To confirm this possibility, following the identification of the gene cluster responsible for linearolide biosynthesis, new derivatives should be created using the genetic engineering methods.

\section{MATERIALS AND METHODS}

Strains, chemicals, and culture conditions

In this study, we used the cytotrienin-producing Streptomyces $\mathrm{sp}$. RK95-74. We used the following producing media: CDY (glucose $2 \%$, soluble starch $1 \%$, meat extract $0.3 \%$, dried yeast $2.5 \%$, corn steep liquor $0.3 \%, \quad \mathrm{~K}_{2} \mathrm{HPO}_{4} \quad 0.05 \%, \quad \mathrm{NaCl} 0.05 \%, \mathrm{CaCO}_{3} \quad 0.05 \%$, $\mathrm{MgSO}_{4} 7 \mathrm{H}_{2} \mathrm{O} 0.05 \%$ ), YMG (malt extract $0.5 \%$, yeast extract $0.5 \%$, glucose $1 \%, \mathrm{CaCO}_{3} 0.1 \%$ ), and $\mathrm{PEC}$ (pectin $2.0 \%$, malt extract $0.5 \%$, casamino acids $0.5 \%$ ). Media were adjusted to a $\mathrm{pH}$ of 6.5 before autoclaving. All chemicals and solvents used were of commercial grade.

\section{HPLC/MS analysis of culture extracts}

The strain RK95-74 was cultured for 4 to 5 days at $28^{\circ} \mathrm{C}$ in cylindrical flasks containing $70 \mathrm{ml}$ of the producing medium (CDY, YMG or PEC). Cultured broth was first extracted with three volumes of
Table 2 Cytotoxicity of linearolides A and B

\begin{tabular}{lcc}
\hline & \multicolumn{2}{c}{$I_{50}(\mu \mathrm{m})$} \\
\cline { 2 - 3 } Cell line & Linearolide $A(\mathbf{1})$ & Linearolide $B(2)$ \\
\hline HL-60 & 6.6 & 91 \\
HeLa & 21 & $>100$ \\
A549 & 20 & $>100$ \\
MCF-7 & 25 & $>100$ \\
Jurkat & 25 & $>100$ \\
U937 & 71 & $>100$
\end{tabular}

acetone, and then twice with an equal volume of ethyl acetate after removal of acetone, and the organic layer was subsequently concentrated in vacuo. Next, $10 \mu \mathrm{l}$ of the extracts dissolved in methanol were then injected into a HPLC system (Waters Alliance 2975 system, Waters PDA 2995 detector, Milford, MA, USA) and connected to MS (Q-trap, Life Technologies, Carlsbad, CA, USA) by using an ODS column (Waters XTerra MS $\phi 2.0 \times 150 \mathrm{~mm}$ ) and aqueous acetonitrile (20-100\% acetonitrile in $30 \mathrm{~min}, 0.2 \mathrm{ml} \mathrm{min}^{-1}$ ).

\section{Production and isolation of new metabolites}

The strain RK95-74 was cultivated with 91 of PEC, YMG and CDY at $28^{\circ} \mathrm{C}$ for 5 days in a $14-1$ jar fermenter (B. E. Marubishi, Co., Ltd., Tokyo, Japan). Resultant culture broths were first extracted with two volumes of acetone and then with equal volume of ethyl acetate twice after removal of acetone. Ethyl acetate extracts (each $100 \mu \mathrm{g} \mathrm{ml}^{-1}$ in methanol, injected $10 \mu \mathrm{l}$ ) were analyzed using HPLC/MS as described above. Peaks specifically identified in YMG and PEC extracts, and not in CDY extract, were targeted for further purification (CDY is the medium used for the production of cytotrienins, the main metabolites of strain RK95-74). Ethyl acetate extracts (1.60 g from YMG, $1.73 \mathrm{~g}$ from PEC, dissolved in chloroform) were fractionated with silica gel chromatography (Silica gel 60, $\phi 40 \times 400 \mathrm{~mm}$, Merck, Darmstadt, Germany) using chloroform and methanol, and divided into 10 fractions. After checking metabolites in each fractions with LC/MS, peaks at $16.6 \mathrm{~min}$ (compound $1: \lambda \max 237 \mathrm{~nm}, \mathrm{~m} / \mathrm{z}$ 808 a.m.u.) and $13.2 \mathrm{~min}$ (compound 2: $\lambda \max 238 \mathrm{~nm}, \mathrm{~m} / \mathrm{z}$ 
782 a.m.u.) were targeted for the further purification. After the peakguided fractionations were performed with HPLC (Waters 660E control system, Waters 2996 PDA detector, and SSC Pegasil-ODS $\phi 20 \times 250 \mathrm{~mm}$ (Senshu Scientific Co., Ltd., Tokyo, Japan) using 40$100 \%$ aqueous acetonitrile at $9 \mathrm{ml} \mathrm{min}^{-1}$ for compound 1 , and $75 \%$ aqueous acetonitrile at $9 \mathrm{ml} \mathrm{min}-1$ for compound 2). In this process, 91 of YMG culture yielded $4.1 \mathrm{mg}$ of compound 1, while 91 of PEC culture yielded $2.3 \mathrm{mg}$ of compound 2 . The structures of these purified compounds were elucidated with various instrumental analyses using JEOL JNM ECA 500 NMR spectrometer, JEOL JMST100LS mass spectrometer (JEOL, Tokyo, Japan). Compounds 1 and 2 were dissolved in methanol- $d_{4}\left(\mathrm{CD}_{3} \mathrm{OD}\right)$ and dimethylsulfoxide- $d_{6}$ $\left(\mathrm{DMSO}-d_{6}\right)$, respectively, for NMR data acquisition.

\section{In vitro antimicrobial and cytotoxic activities}

For in vitro antimicrobial and cytotoxic assays, compounds $\mathbf{1}$ and 2 were dissolved in DMSO. For the antimicrobial assay, each solution was placed on a paper disc $(6 \mathrm{~mm}$ of diameter, TOYO paper Co. Ltd, Tokyo, Japan) to a final concentration of $100 \mu \mathrm{g}$ per disc. These discs were then placed on the agar plates containing test microorganisms, Escherichia coli HO, Pseudomonas aeruginosa L, Staphylococcus aureus 209, Saccharomyces cerevisiae JCM7255 and Candida albicans JCM1542. Agar medium contained 3\% bouillon and 2.4\% potato dextrose for bacterial and fungal growth, respectively.

Human promyelocytic leukemia cells HL-60 (in RPMI1640 + 10\% FBS), human cervix epidermoid carcinoma cells HeLa (in DMEM $+10 \%$ FBS), human adenocarcinomic alveolar basal epithelial cells A549 (in DMEM + 10\% FBS), human breast adenocarcinoma cells MCF-7 (in RPMI1640+10\% FCS), human T-cell leukemia cells Jurkat (in RPMI1640 + 10\% FBS) and human leukemic monocyte lymphoma cells U937 (in RPMI1640 + 10\% FBS) were used in cytotoxic assays. All culture media were supplemented with $50 \mathrm{unit} \mathrm{ml}^{-1}$ of penicillin and $50 \mu \mathrm{g} \mathrm{ml}^{-1}$ of streptomycin. Cells $\left(1 \times 10^{4}\right.$ cells $/ 100 \mu$ l per well $)$ were seeded on a 96 -well microtiter plates (IWAKI, Asahi Glass Co., Ltd., Tokyo, Japan) and incubated for $24 \mathrm{~h}$ at $37^{\circ} \mathrm{C}$ in a $\mathrm{CO}_{2}$ incubator. After a 48 -h challenge with serially diluted compound solutions, cytotoxic effects were determined with a modified MTT colorimetric method using WST-8 (Nacalai tesque, Kyoto, Japan). $\mathrm{IC}_{50}$ values were estimated from graphic plots.

\section{ACKNOWLEDGEMENTS}

This work was supported by the Program for Promotion of Basic and Applied Researches for innovations in Bio-oriented Industry. This study was also supported by a KAKENHI grant (Grants-in-Aid for Scientific Research) from the Ministry of Education, Culture, Sports, Science and Technology, Japan.

1 Kieser, T., Bibb, M. J., Buttner, B. J., Chaterm, K. F. \& Hopwood, D. A. Practical Streptomyces Genetics (John Innes Foundation, Norwich, 2000).

2 Kurtboke, D. I. Biodiscovery from rare actinomycetes: an eco-taxonomical perspective. Appl. Microbiiol. Biotechnol. 93, 1843-1852 (2012).

3 Newman, D. J. \& Cragg, G. M. Natural products as sources of new drugs over the 30 years from 1981 to 2010. J. Nat. Prod. 75, 311-335 (2012).

4 Bentley, D. S. Complete genome sequence of the model actinomycete Streptomyces coelicolor A3(2). Nature 417, 141-147 (2002).

$5 \mathrm{Ikeda}, \mathrm{H}$. et al. Complete genome sequence and comparative analysis of the industrial microorganism Streptomyces avermitilis. Nat. Biotechnol. 21, 526-531 (2003).

6 Nett, M., H. Ikeda, H. \& Moore, B. S. Genomic basis for natural product biosynthetic diversity in the actinomycetes. Nat. Prod. Rep. 26, 1362-1384(2009).

7 Chen, Y. et al. A proteomic survey of nonribosomal peptide and polyketide biosynthesis in Actinobacteria. J. Proteome Res. 11, 85-94 (2012).

8 Wilkinson, B. \& Micklefield, J. Mining and engineering natural-product biosynthetic pathways. Nat. Chem. Biol. 3, 379-386 (2007).

9 Zhang, H.-P., Kakeya, H. \& Osada, H. Novel triene-ansamycins, cytotrienins A and B, inducing apoptosis on human leukemia HL-60 cells. Tetrahed. Lett. 38, 1789-1792 (1997).

10 Kakeya, H. et al. Cytotrienin A, a novel apoptosis inducer in human leukemia HL-60 cells. J. Antibiot. 50, 370-372 (1997).

11 Lee, W. W., Wu, H. Y., Christensen, J. E., Goodman, L. \& Henry, D. W. Confirmation by synthesis of the structure of acosamine and methyl $\mathrm{N}$-acetylactinosaminide. J. Med. Chem. 18, 768-769 (1975).

\footnotetext{
(c) (i) () $\Theta$ This work is licensed under a Creative Commons Attribution-NonCommercial-NoDerivs 3.0 Unported License. To view a copy of this license, visit http://creativecommons. org/licenses/by-nc-nd/3.0/
}

Supplementary Information accompanies the paper on The Journal of Antibiotics website (http://www.nature.com/ja) 